\title{
Etude systématique, habitats et répartion géographique des Setodes (Trichoptera : Leptoceridae) de Madagascar
}

\author{
D. Randriamasimanana ${ }^{1}$ \\ F.-M Gibon ${ }^{2}$
}

Mots clés : Trichoptera, Leptoceridae, Leptocerinae, Setodes, nouvelles espèces, morphologie, géographie, écologie, Madagascar.

Le genre Setodes est signalé pour la première fois à Madagascar. Cinq espèces nouvelles sont décrites. Les affinités de ce petit groupe relativement homogène sont asiatiques et l'hypothèse d'une colonisation probable de la Grande Île depuis l'Inde est discutée. La répartition géographique et l'écologie des espèces sont analysées en fonction de l'altitude et de la distance de la source.

A systematic study, habitats and geographic distribution of Setodes (Trichoptera : Leptoceridae) from Madagascar

Keywords : Trichoptera, Leptoceridae, Leptocerinae, Setodes, new species, morphology, Madagascar.

The genus Setodes is reported for the first time from Madagascar. Five new species are described. The relationships of this small homogeneous group are asiatic. The hypothesis of a colonisation of the island from India is discussed. An account of the geographic distribution of the five species is given and in relation to altitude and distance to the source.

\section{Introduction}

Au cours d'un programme d'étude de la faune aquatique des eaux douces malgaches, un peu plus de quatre cents sites ont été inventoriés au moyen de pièges lumineux. Les objectifs et méthodes de ce programme ont été précédemment exposés (Gibon \& Elouard 1996). Cinq espèces endémiques appartenant au genre Setodes ont été capturées : $S$. reynae n. sp., $S$. madagasca n. sp., S. fabienneae n. sp., S. heryae n. sp. et $S$. orientalis $\mathrm{n}$. sp.

\section{Taxinomie}

Les Setodes malgaches constituent un petit groupe homogène. Ils sont de petite taille, d'allure mince et frêle ; les ailes sont pointues et étroites. La coloration est brun pâle. La nervation alaire varie peu d'une espè-

1. Université d'Antananarivo / IRD, B.P. 434, Antananarivo, Madagascar.

2. 160 chemin des processions, $F-34820$ Assas. ce à l'autre. Aux ailes antérieures, la cellule thyridiale est beaucoup plus longue que la cellule discoïdale. La nervule transverse radiale est insérée avant la base de la furca 1 . De même, la nervule médiane est insérée avant la furca 3 (sauf chez $S$. orientalis, où cette furca est presque sessile). La nervule radio-médiane est située avant les nervules radiale et médiane. Les nervules radio-médiane et médiane sont obliques, leur inclinaison est opposée à celle de la nervule radiale. Aux ailes postérieures, la base de la radiale a disparu, elle apparaît un peu avant la nervule radio-médiane (ceci constitue l'un des caractères fondamentaux du genre Setodes). La furca M1+2/M3+4 est petite et longuement pétiolée.

2.1. Setodes reynae Randriamasimanana \& Gibon n. sp.

(Figs. 1 à 5)

\section{Description}

La longueur de l'aile antérieure est de 4,6 mm, celle de l'aile postérieure de $3,7 \mathrm{~mm}$. 


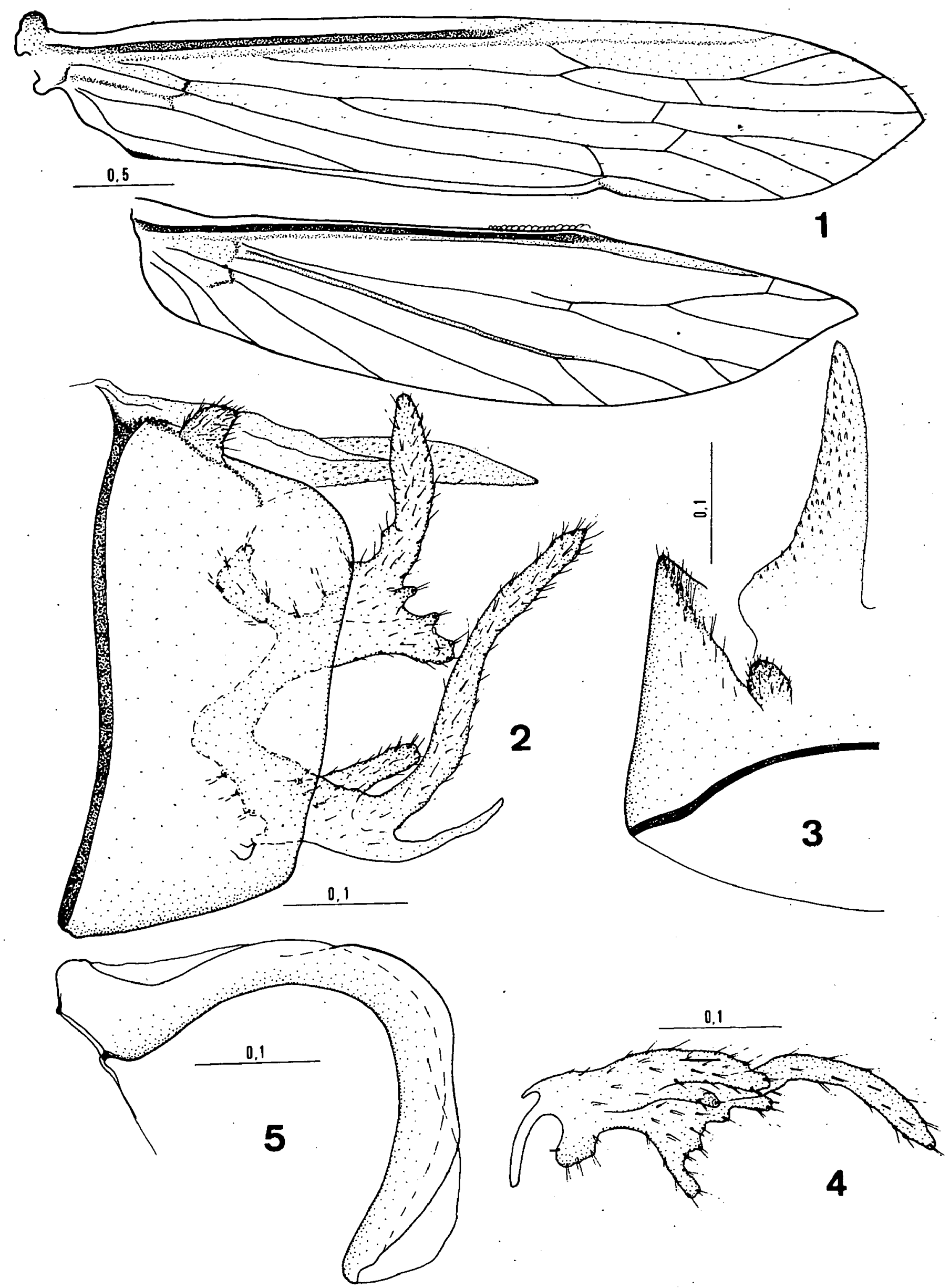

Figs. 1 à 5. Setodes reynae n. sp. 1. ailes antérieure et postérieure, 2. vue latérale des genitalia mâles, 3. vue dorsale partielle des genitalia mâles, 4 . vue ventrale d'un appendice inférieur, 5 . appareil phallique en vue latérale. Echelle en mm.

Figs. 1 to 5. Setodes reynae sp. $n$. 1: fore and hindwing, 2. lateral view of the male genetalia, 3. partial dorsal view of the male genitalia, 4. ventral view of an inferior appendage, 5. lateral view of the phallic apparatus. Scale : $\mathrm{mm}$. 


\section{Genitalia mâles}

En vue latérale, le neuvième segment abdominal est bien développé, sub-rectangulaire en vue latérale. Les appendices préanaux sont petits et globuleux, en forme de petit bouton en vue latérale et ovoïdes en vue dorsale. Le dixième segment abdominal est constitué d'un seul élément. Il est profondément divisé en deux lobes couverts de nombreuses petites épines en forme d'écaille pointue. En vue latérale, il apparaît comme une plaque courte, épaisse et horizontale ; tandis qu'en vue dorsale, il est bifide, très large à la base et pointu à l'apex. Les appendices inférieurs ont une forme très complexe et caractéristique. Ils constituent l'élément le plus apparent des genitalia, bien que leur partie basale soit située à l'intérieur du neuvième segment abdominal. En vue latérale, ils sont constitués par deux branches. Dès la base, chaque appendice est divisé en une plaque ventrale et une branche dorsale. La branche dorsale, très haute, puisqu'elle dépasse le bord dorsal du dixième segment, est pourvue, à mi-hauteur, de deux lobes secondaires, l'un proximal, l'autre distal lui donne un vague profil en croix. La plaque ventrale est réduite, elle donne naissance à une branche dorsale secondaire longue et digitiforme ainsi qu'à divers petits lobes secondaires sur sa face interne. L'appareil phallique est simple, élégamment courbé et dépourvu de tout sclérite interne.

Holotype : mâle capturé le 4/04/96 à Ambohimanatrika sur la rivière Kamoro (bassin du fleuve Betsiboka ; station St02-38, long. 47:10:06 E, lat. 16:28:55 S, alt. $40 \mathrm{~m}$ ). Il est référencé P0578-2. Il se compose de deux préparations sur lame, l'une dans l'Euparal (genitalia avec l'appareil phallique) et l'autre à sec (ailes antérieure et postérieure). Le reste du corps est conservé dans l'alcool. Il est déposé au Laboratoire d'Entomologie du M.N.H.N. à Paris.

Paratypes : 20 paratopotypes mâles, déposés au C.N.R.E (Antananarivo).

Etymologie : nous dédions cette espèce à Reyna $\mathbf{R a}$ biaza.

\subsection{Setodes madagasca Randriamasimanana \& Gibon} n. sp.

(Figs. 6 à 10)

\section{Description}

La longueur de l'aile antérieure est de $4,7 \mathrm{~mm}$, celle de l'aile postérieure de 3,9 mm.

\section{Genitalia mâles}

En vue latérale, le neuvième segment abdominal est

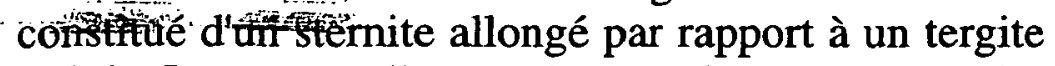
réduit. Les appendices préanaux forment de petits lobes apparaissant triangulaires en vue latérale, mais ovoïdes en vue dorsale. Le dixième segment abdominal est constitué d'un seul lobe, courbé ventralement et muni de nombreuse petites épines en forme d'écaille. Il est profondément divisé dans le sens de la longueur ; chaque branche latérale est courbée ventralement et régulièrement amincie jusqu'à une pointe distale. La base est surmontée dorsalement par une bosse saillante. Les appendices inférieurs sont très développés, avec une base partiellement cachée dans le neuvième segment. Ils sont formés de deux branches presque perpendiculaires. L'une, dorsale, est massive en vue latérale, munie sur la face interne de petits tubercules portant quelques soies sommitales. L'autre, qui prolonge distalement la base, est fourchue à partir de la mi-longueur ; la branche supérieure est plus courte et plus fine que la branche inférieure horizontale. L'appareil phallique est simple, dépourvu de sclérites internes.

Holotype : mâle capturé le 12/11/96 à Andranomaitso sur la rivière Vintanona (bassin du fleuve Mananjary ; station St18-11, long. 47:45:14 E, lat. 21:20:37 S, alt. $320 \mathrm{~m}$ ). Il est référencé P0650-3. Il se compose de deux préparations sur lame, l'une dans l'Euparal (genitalia avec appareil phallique) et l'autre à sec (ailes antérieure et postérieure). Le reste du corps est conservé dans l'alcool. Il est déposé au Laboratoire d'Entomologie du M.N.H.N. à Paris.

Paratypes : 20 paratopotypes mâles, déposés au C.N.R.E (Antananarivo).

Etymologie : d'après la traduction française du nom de lî̀le, Madagascar.

\subsection{Setodes fabienneae Gibon \& Randriamasimanana n. sp.}

(Figs. 11 à 15)

\section{Description}

La longueur de l'aile antérieure est de $5,1 \mathrm{~mm}$, celle de l'aile postérieure de $4,5 \mathrm{~mm}$.

\section{Genitalia mâles}

Le neuvième segment abdominal est large, sauf au niveau du tergite qui est réduit dorsalement. Les appendices préanaux sont courts et globuleux. Le dixième segment abdominal est réduit à un seul lobe profondément divisé en deux branches latérales, comme chez les deux espèces précédentes. Cependant il est moins développé, les branches distales sont plus fines et moins allongées. Sa partie supérieure porte une petite bosse dorso-distale semblable à celle décrite chez $S$. madagasca. En revanche, les appendices inférieurs sont plus développés. La base est cachée dans le neuvième segment abdominal et donne naissance à trois 


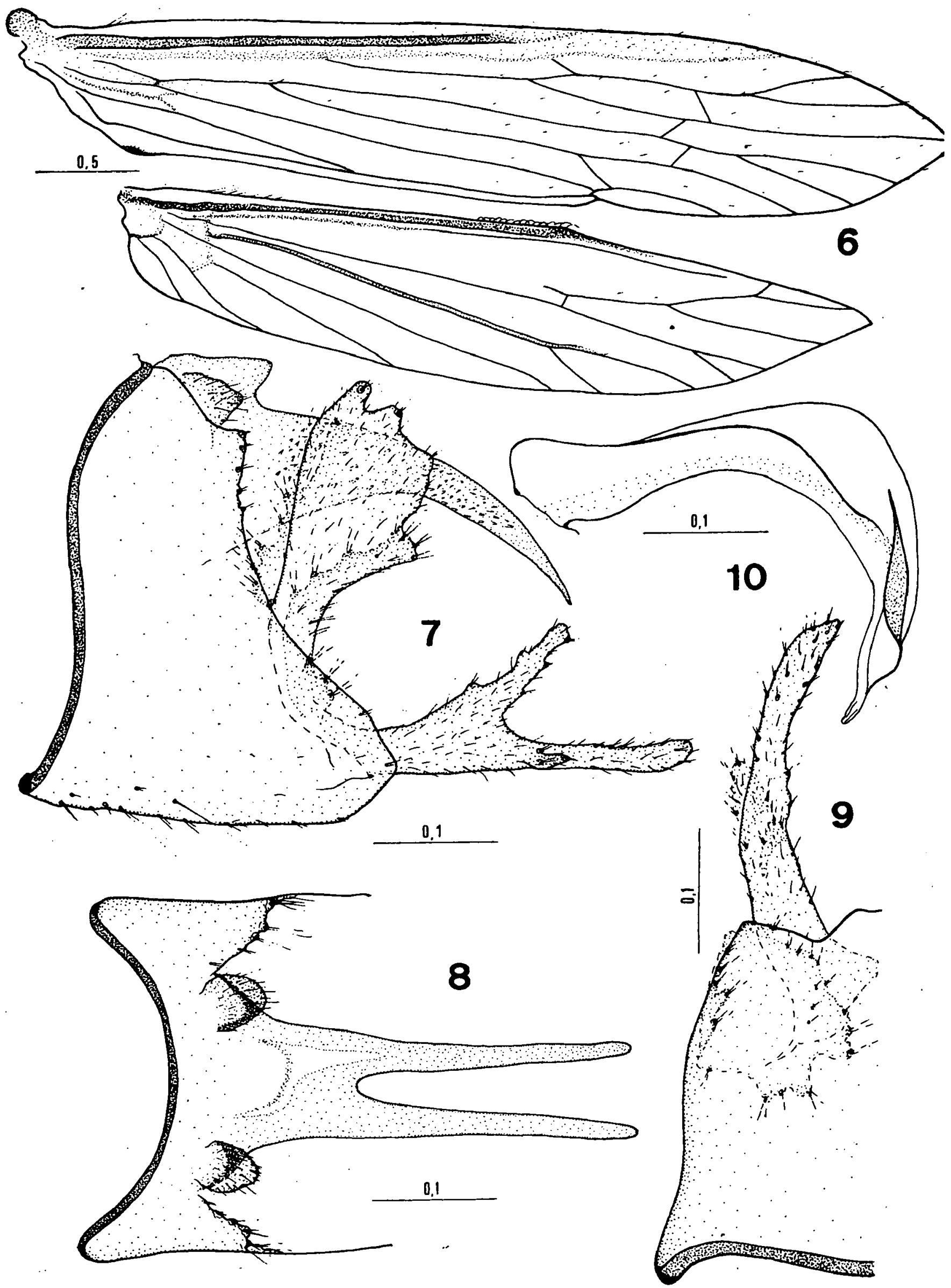

Figs. 6 à 10. Setodes madagasca n. sp. 6. ailes antérieure et postérieure, 7. vue latérale des genitalia mâles, 8. vue dorsale des genitalia, 9. vue ventrale partielle, 10. appareil phallique en vue latérale. Echelle en mm.

Figs. 6 to 10. Setodes madagasca sp. n. 6 . fore and hindwing, 7. lateral view of the male genitalia, 8. dorsal view of the genitalia, 9. partial ventral view, 10. lateral view. of the phallic apparatus. Scale : $\mathrm{mm}$. 

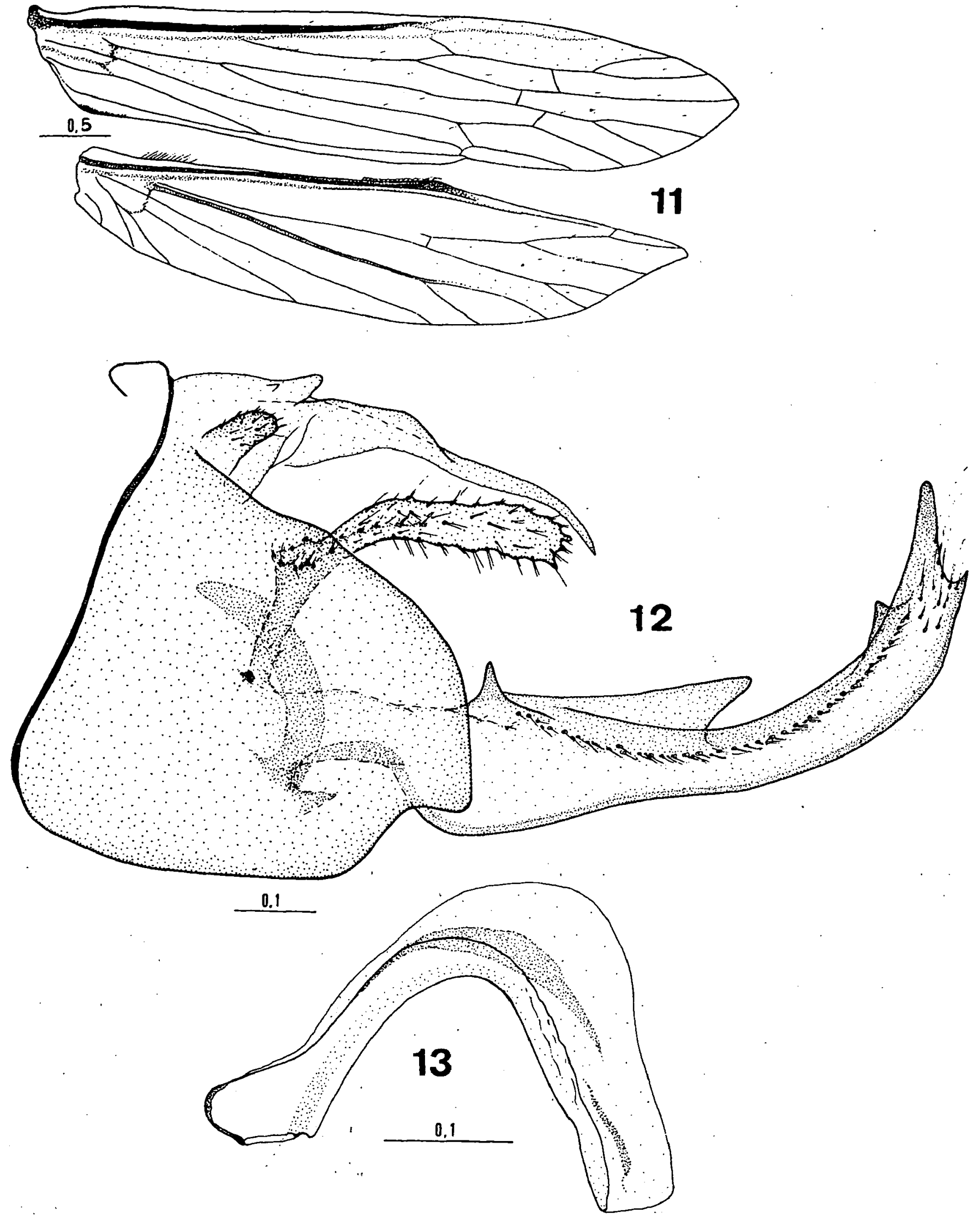

Figs. 11 à 13. Setodes fabienneae $\mathrm{n}$. sp. 11. ailes antérieure et postérieure, -12 : vue latérale dès-genitalia-mâles,-13. appareil phällique en vue lätérále. Echelle en $\mathrm{mm}$

Figs. 11 to 13. Setodes fabienneae sp. n. 11. fore and hindwing, 12. lateral view of the male genitalia, 13. lateral view of the phallic apparatus. Scale : $\mathrm{mm}$. 

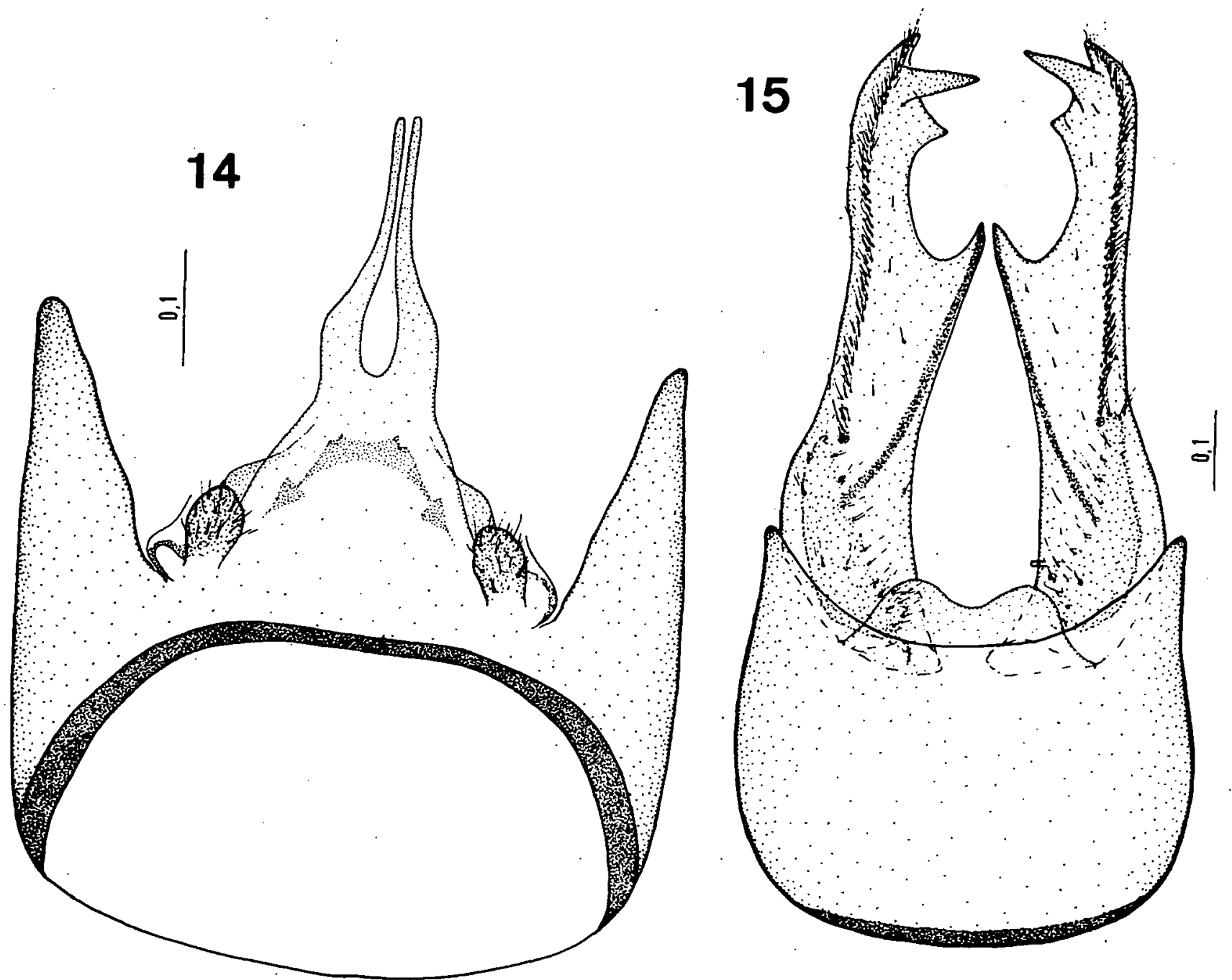

Figs. 14 à 15. Setodes fabienneae n. sp. 14. vue dorsale du dixième segment, 15. vue ventrale des appendices inférieurs. Echelle en mm

Figs. 14 to 15 . Setodes fabienneae sp. n. 14. dorsal view of the tenth segment, 15. ventral view of the inferior appendages. Scale : $\mathrm{mm}$.

branches de formes et de tailles différentes. La branche distale, très allongée, massive et fortement sclérotisée, est munie d'une ligne des soies sur la face externe ainsi que de carènes qui forment quelques pointes saillantes sur les faces supérieures et internes. Les deux branches dorsales sont plus réduites. La première, située en position externe et orientée dorso-distalement, est en forme de massue, courbée ventralement et couverte de petites soies. La seconde, interne et moins apparente, est digitiforme et nettement sclérotisée. L'appareil phallique est simple ; la phallothèque est un peu plus large que chez les espèces précédentes.

Holotype : mâle capturé le 17/04/94 à $19 \mathrm{~km}$ de Ranohira, sur la rivière Menamaty (bassin du fleuve Mangoky ; station. St01-13, long. 45:23:46 E, lat. 22:25:50 S, alt. $650 \mathrm{~m}$ ). Il est référencé P0300-4. Il est composé de deux préparations sur lame, l'une dans l'Euparal (genitalia avec appareil phallique), l'autre à sec (ailes antérieure et postérieure). Le reste du corps est conservé dans l'alcool. Il est déposé au Laboratoire d'Entomologie du M.N.H.N. à Paris.

Paratypes : 30 paratopotypes mâles, déposés au C.N.R.E (Antananarivo).

Etymologie : nous dédions cette espèce à Fabienne Ranaivoharindriaka.

2.4. Setodes heryae Randriamasimanana \& Gibon $\mathrm{n}$. sp. (Figs. 16 à 20)

\section{Description}

La longueur de l'aile antérieure est de $5,3 \mathrm{~mm}$, celle de l'aile postérieure de $4,4 \mathrm{~mm}$.

Genitalia mâles

Le neuvième segment abdominal est long surtout au niveau des appendices inférieurs. Le tergite est très réduit, ce qui donne au segment un profil quasi-triangulaire. Les appendices préanaux sont en forme de petit 

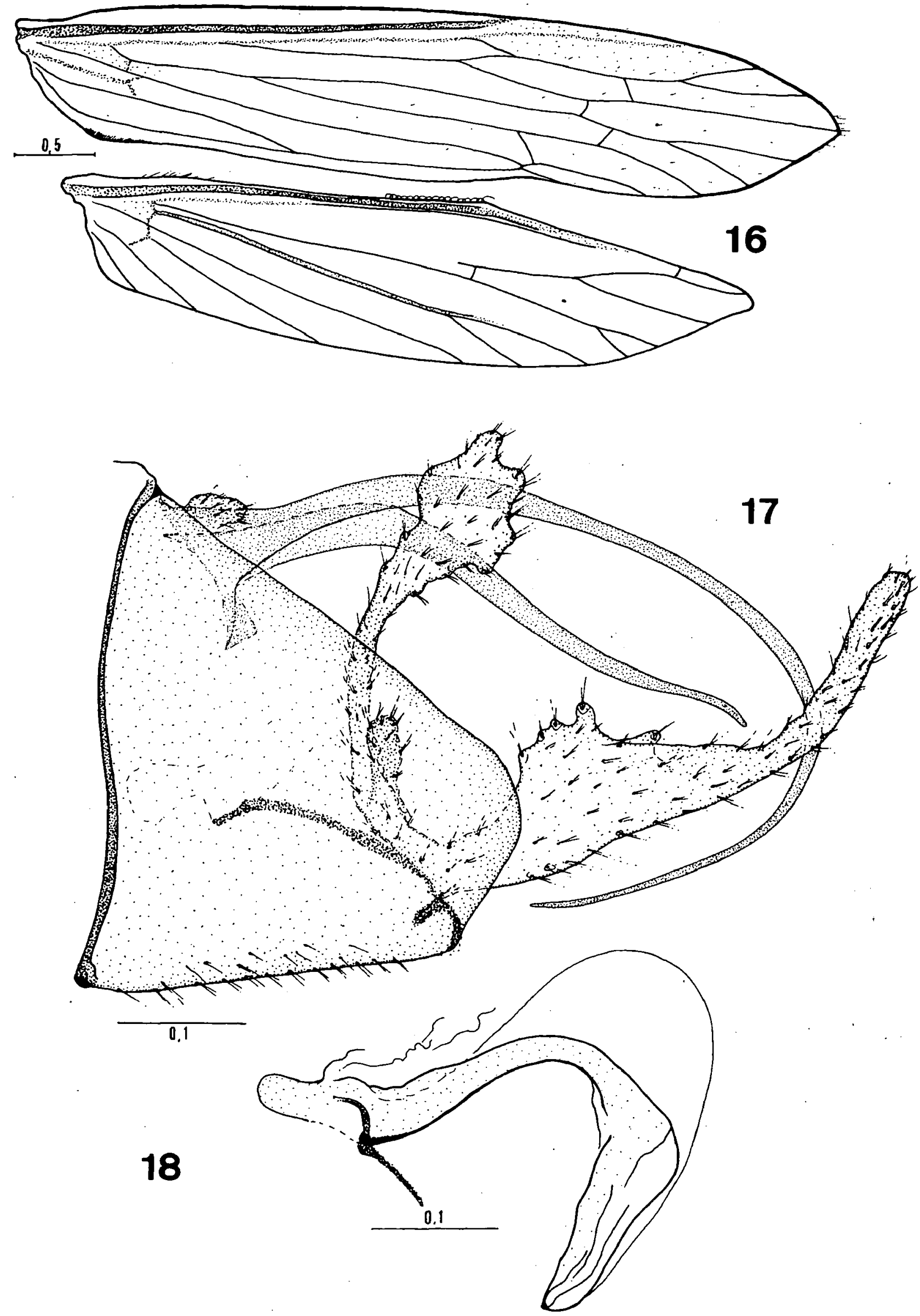

Figs. 16 à 18. Setodes heryae n. sp. 16. ailes antérieure et postérieure, 17. vue latérale des genitalia mâles, 18. appareil phallique en vue latérale. Echelle en $\mathrm{mm}$.

Figs. 16 to 18 . Setodes heryae sp. n. 16. fore and hindwing, 17. lateral view of the male genitalia, 18. lateral view of the phallic apparatus. Scale : $\mathrm{mm}$. 

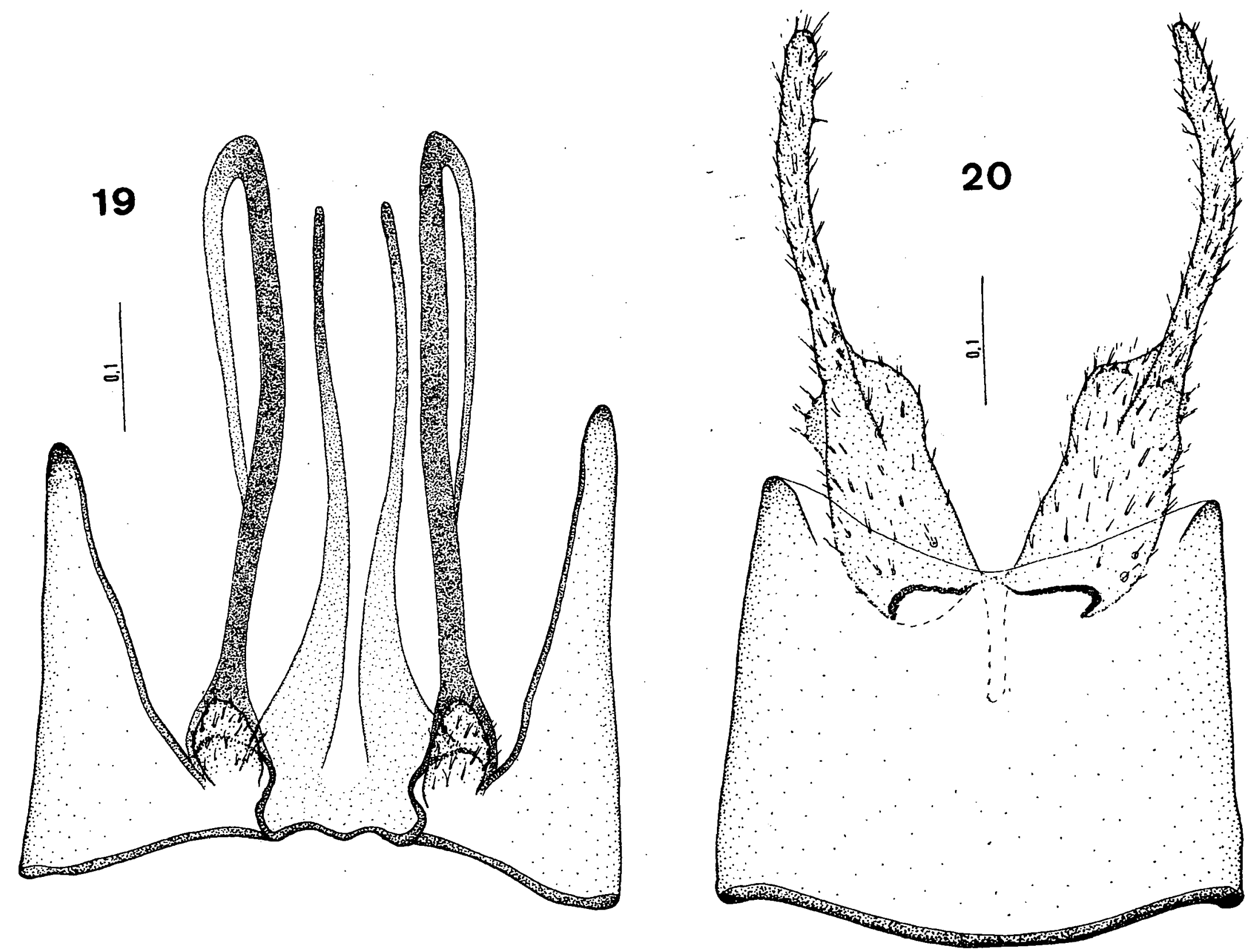

Figs. 19 à 20. Setodes heryae n. sp. 19. vue dorsale du dixième segment, 20. vue ventrale des appendices inférieurs. Echelle en mm.

Figs. 19 to 20. Setodes heryae sp. $n$. 19. dorsal view of the tenth segment. 20. ventral view of the inferior appendage. Scale : mm.

lobe. Le dixième segment abdominal est plus transformé que chez les autres espèces. La base est réduite et peu visible; la séparation en deux parties latérales symétriques est plus prononcée. De plus, chacune de ces parties latérales est elle-même secondairement divisée en deux branches. L'une, dorsale, externe et très longue, présente une courbure semi-elliptique en vue latérale. L'autre, ventro-interne, est plus courte ; sa longueur n'atteint que les deux tiers de celle de la précédente. Elle est légèrement sinueuse et dirigée ventrodistalement. Les appendices inférieurs, constitués de deux branches presque perpendiculaires, sont semblables à ceux de $S$. madagasca. Cependant, la branche verticale, moins large, présente, vers la base et en position interne, un lobule secondaire digitiforme. La branche horizontale n'est pas fourchue ; sa moitié ba- sale élargie est visible autant en vue ventrale que latérale ; la moitié distale est digitiforme. L'appareil phallique est simple.

Holotype : mâle capturé le 19/04/93 sur une mare près du village de Voandelaka (bassin du fleuve Fiherenana ; station St16-05, long. 44:35:03 E, lat. 22:51:35 S, alt. $530 \mathrm{~m}$ ). Il est référencé P0134-2. Il est composé de deux préparations sur lame, l'une dans l'Euparal (genitalia avec appareil phallique), l'autre à $\mathrm{sec}$ (ailes antérieure et postérieure). Le reste du corps est conservé dans l'alcool. Il est déposé au Laboratoire d'Entomologie du M.N.H.N. à Paris.

Paratype : un paratopotype mâle, déposé au C.N.R.E (Antananarivo).

Etymologie : cette espèce est dédiée à Hery Ranarijaona. 


\subsection{Setodes orientalis Randriamasimanana \& Gibon n. sp.}

(Figs. 21 à 25)

\section{Description}

Cette espèce présente une caractéristique antennaire remarquable : les articles 2 à 11 sont raccourcis et en forme d'anneaux. Une autre particularité est l'insertion supérieure de la nervule médiane des ailes antérieures à la base de la furcation de la médiane et non pas en deçà comme chez les autres Setodes malgaches. La longueur de l'aile antérieure est de 4,2 $\mathrm{mm}$, celle de l'aile postérieure de $3,6 \mathrm{~mm}$.

\section{Genitalia mâles}

Le neuvième segment abdominal est large, avec un renflement latéral au niveau de l'insertion des appendices inférieurs. Le tergite est dorsalement réduit. Les appendices préanaux sont en forme de bouton; ils sont plus petits que chez les autres espèces. Le dixième segment abdominal est composé d'un seul élément profondément divisé en deux branches latérales fortement sclérotisées. Celle-ci sont d'abord dirigées distalement, fortement courbées ventralement, puis la courbure s'inverse légèrement vers l'apex. La base des appendices inférieurs est cachée dans le neuvième segment abdominal. Ces appendices présentent la structure décrite chez les autres Setodes malgaches. Une branche distale très allongée donne naissance vers le tiers de sa longueur à un lobe secondaire digitiforme dirigé dorsalement. Une branche dorsale, nettement plus petite et moins élargie que chez les autres espèces, porte également des lobules secondaires. En vue ventrale, les deux appendices sont en forme de pince, dont les extrémités sont arquées l'une vers l'autre. L'appareil phallique est simple mais plus sclérotisé que chez les autres espèces ; il apparaît comme un tube courbé ventralement.

Holotype : mâle capturé le 15/04/97 à Sarahimbola, sur le fleuve Ivoloina (station St 107-06, long. 49:21:05 $\mathrm{E}$, lat. 18:02:02 S, alt. $30 \mathrm{~m}$ ). Il est référencé P0691-2. Il est composé de deux préparations sur lame, l'une dans l'Euparal (genitalia avec appareil phallique), l'autre à sec (ailes antérieure et postérieure). Le reste du corps est conservé dans l'alcool. Il est déposé au Laboratoire d'Entomologie du M.N.H.N. à Paris.

Paratype : 1 paratopotype mâle, déposé au C.N.R.E (Antananarivo).

Etymologie : le nom a été choisi en fonction de la localisation de cette espèce sur la côte orientale.

\section{Remarques sur la phylogénie du groupe}

Bien qu'il n'ait pas souhaité l'intituler "révision", le travail de Schmid (1987) sur les Setodes constitue une remarquable synthèse de ce genre. Il y démontre, qu'en l'état actuel de nos connaissances, la phylogénie du genre ne peut être exactement reconstituée. En revanche, il y distingue cinq grandes lignées : la "Branche Primitive", les "Groupes et Espèces Isolés", les "Espèces Blanches", les "Espèces Bouclées" et les "Espèces Etranges". Ces lignées sont elles-même divisées en groupes d'espèces. Elles ne correspondent pas à des taxons formellement établis mais leur réalité phylogénique n'est pas contestable. Cette partition permet une bien meilleure approche de la Systématique et de lá Biogéographie d'un genre très largement répandu et riche de plus de deux cents espèces décrites. Ce qui complique un peu la situation, c'est que trois genres, actuellement reconnus et bien caractérisés, s'intègrent dans cet ensemble. Il s'agit de Sericodes et d'Hemileptocerus, deux petits genres africains qui sont proches des Groupes et Espèces Isolés, mais surtout de Trichosetodes, genre asiatique et africain, voisin des Espèces Etranges. Setodes n'est donc pas un genre monophylétique. Trichosetodes s'est différencié de Setodes après que ce dernier se soit divisé en cinq branches. Il est très voisin des Espèces Etranges, dont il représente.un rameau spécialisé

Les cinq espèces malgaches, décrites ci-dessous constituent un groupe (sensu Schmid) homogène. Par certains aspects, il est très proche des Trichosetodes africains. Ces derniers présentent une petite taille, une allure délicate et une coloration brun clair ; leurs ailes sont plus étroites, plus longues et plus effilées qu'il n'est de règle chez les Setodes. Tous ces caractères sont partagés par le groupe des Setodes malgaches. Pour d'autres, la situation est ambigüe. La plus grande largeur de l'aile antérieure est située au niveau de l'extrémité de la cellule discoïdale chez les Setodes, et vers le milieu de l'aile chez les Trichosetodes. Dans le groupe malgache, la situation est intermédiaire, la largeur restant à peu près constante sur une grande partie de l'aile. Le genre Trichosetodes est facilement identifiable grâce à la présence d'un pinceau de soies à l'extrémité du scape ; ce caractère est absent chez les Setodes malgaches, mais il l'est également chez deux Trichosetodes d'Asie orientale (le groupe polonorum, sensu Schmid). C'est donc au niveau des genitalia mâles que se situe le principal clivage entre les Setodes malgaches et les Trichosetodes. Chez ces derniers, le dixième segment abdominal est représenté par une ou deux paires de branches latérales nettement séparées, la partie basale a disparu ou est vestigiale. Chez les $\mathrm{Se}$ - 

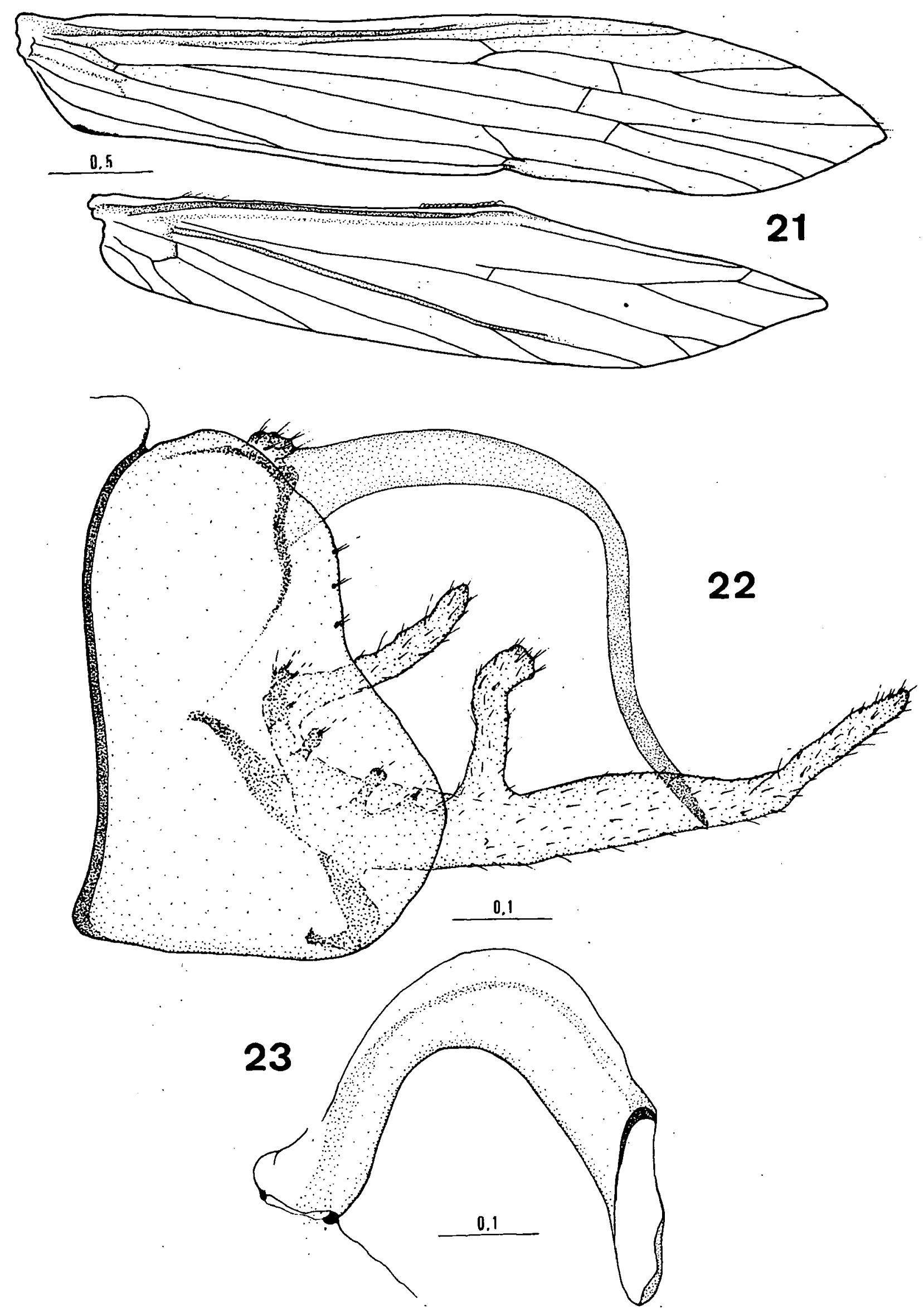

Figs. 21 à 23. Setodes orientalis n. sp. 21. ailes antérieure et postérieure, 22. vue latérale des genitalia mâles, 23, appareil phallique en vue latérale. Echelle en mm.

Figs. 21 to 23 . Setodes orientalis sp. n. 21. fore and hindwing, 22. lateral view of the male genitalia, 23. lateral view of the phallic apparatus. Scale : $\mathrm{mm}$. 

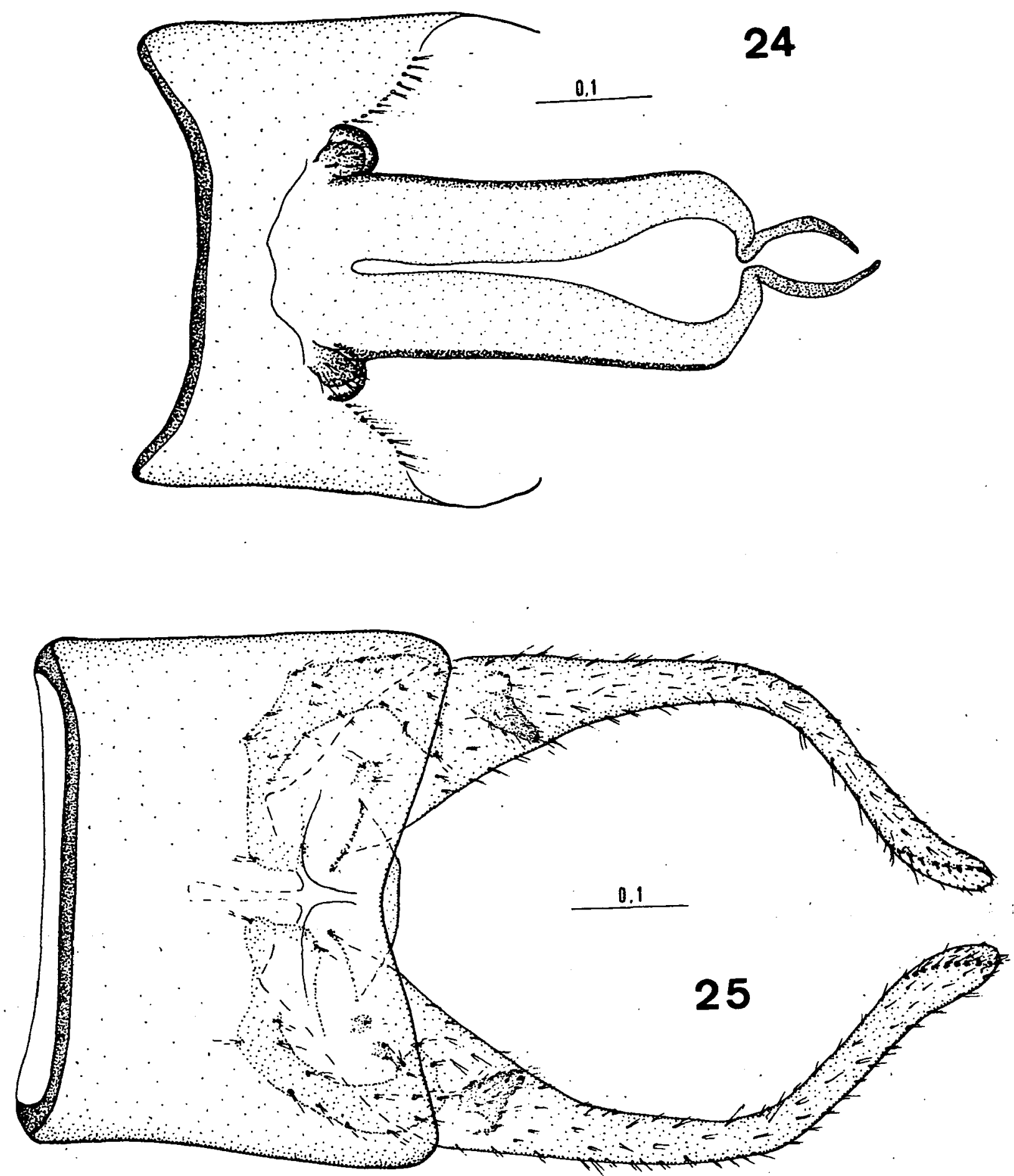

Figs. 24 à 25 . Setodes orientalis $\mathbf{n}$. sp. 24. vue dorsale du dixième segment,'25. vue ventrale des appendices inférieurs. Echelle en mm.

Figs. 24 to 25 . Setodes orientalis sp. n. 24. dorsal view of the tenth segment, 25. ventral view of the inferior appedages. Scale : $\mathrm{mm}$.

todes malgaches, le dixième segment est divisé en deux éléments latéraux plus ou moins allongés, mais la partie basale est présente et souvent bien développée. On notera également les appendices préanaux souvent en forme de bouton ou de petite bosse et non allongés comme chez-Trichosetodes. On notera enfin que l'appareil phâliqué n'est pas aussi fortement et uniformément sclérotisé.
Dans l'arbre phylétique élaboré par Schmid, les $S e$ todes malgaches se situent donc entre la branche des Espèces Etranges et le genre Trichosetodes; nous pouvons les supposer proches de ce qu'ont pu être les Trichosetodes ancestraux. Les implications biogéographiques, concernant l'origine du peuplement de la Grande Île, sont intéressantes. En effet, l'apparition, l'évolution et la spéciation des Setodes ont eu lieu en 
Asie. L'Afrique a été colonisée par plusieurs branches, mais pas par celle des Espèces Etranges. En revanche, elle a été colonisée par le genre Trichosetodes, mais pas par les formes les plus archaïques (le groupe polonorum d'Extrême Orient). Aucune des lignées communes à l'Asie et à l'Afrique n'est présente à Madagascar. Les affinités des Setodes malgaches sont asiatiques (même en tenant compte d'une parenté certaine avec les Trichosetodes), plus particulièrement indiennes. L'hypothèse la plus simple est donc celle d'une colonisation de la Grande Ile à partir du souscontinent indien.

Cette question de l'origine asiatique de certains éléments de la faune malgache est ancienne. L'hypothèse d'un corridor terrestre a été émise par Steenis en 1962, pour expliquer des distributions d'espèces souvent voisines, peuplant Madagascar, l'Inde ou l'Asie du SudEst. D'après ce que l'on sait maintenant de l'histoire géologique de l'océan indien (Mckenzie \& Sclater 1973), un tel pont n'a jamais existé. Mais, pour de nombreux groupes le passage a bien eu lieu (Rage' \& Jaeger 1995). D'après Beck et al. (1995) une connexion aurait été établie entre l'Inde et l'Asie vers la fin du Crétacé, hypothèse fondée sur des arguments géologiques et géophysiques. A cette époque, le bloc seychellois constitue une passerelle entre l'Inde et Madagascar, et l'on ne peut exclure la possibilité de migrations "step by step", du continent asiatique vers la Grande Ile. Pour Schatz (1996), il est plus probable que ces migrations aient eu lieu à l'Oligocène, lors de la régression maritime qui suivit la rencontre du bloc indien et de l'Asie. A cette époque, il est probable que les parties hautes des plateaux de Chagos et des Seychelles aient été émergées, elles ont pu constituer une voie de migration de l'Inde vers Madagascar, qui a été appelée "Lemurian stepping-stones". Cette hypothèse d'une route terrestre en pointillés entre l'Asie et Madagascar, qui aurait été ouverte à une date que l'on ne peut encore situer exactement entre la fin du Crétacé et l'Eocène, explique de nombreuses anomalies de distribution, aussi bien animales (Rage, 1996) que végétales (Schatz, 1996).

\section{Habitats et répartition géographique}

Pour le genre Setodes, puis pour chacune des espèces, nous avons établi une carte des localités de capture ainsi que leurs profils écologiques. Ces derniers sont établis à partir de deux indicateurs écologiques fondamentaux : l'altitude (ordonnée) et la distance de la source (abscisse) (Fig. 26). Pour prendre en considération le facteur dominant de la biogéographie malgache, la distinction entre les versants oriental et occidental
(Battistini 1996), les distances de la source sont figurées positivement pour les bassins orientaux et négativement pour les bassins occidentaux. Cette méthode est une adaption de celle utilisée par Décamps (1968) ; elle a été présentée et discutée par Gibon (2000).

La figure 27 indique le profil écologique de l'ensemble des stations échantillonnées, ainsi que celui des sites de capture du genre Setodes. Bien qu'il ne comprenne que cinq espèces, Setodes est très répandu à Madagascar, puisqu'il est capturé sur $52 \%$ des 404 sites que nous avons étudiés. Il colonise surtout les moyennes et grandes rivières, la majorité des sites négatifs sont situés à moins de 10 kilomètres des sources.

- S. madagasca (figure 28) est l'espèce la plus commune. Elle a été capturée sur 142 stations (chez les Trichoptères, seul Macrostemum graphicum est plus fréquent avec 194 captures). Ces stations sont réparties sur presque toute l'île, à la fois sur les bassins occidentaux (Betsiboka, Tsiribihina, Mangoky, Onilahy, Menarandra et Fierenana) et orientaux (Mangoro, Rianila, Namorona, Sakanila, Manampatrana, Mananjary et Matitanana). Elle occupe toute la gamme altitudinale et toute celle des distances aux sources. Elle est rare dans deux cas : d'une part, les petites rivières (- $10 \mathrm{ki}$ lomètres des sources), d'autre part, les basses altitudes du versant occidental (au-dessous de $1.000 \mathrm{~m}$ ). A l'exception de quelques rares captures de $S$. reynae, $S$. madagasca est la seule espèce présente au-dessus de $1.000 \mathrm{~m}$ sur le versant occidental (région des HautesTerres centrales), c'est également la seule présente audessus de $250 \mathrm{~m}$ sur le versant oriental.

- $S$. reynae (Fig. 27) est également une espèce fréquente et largement distribuée. Elle est capturée sur les bassins occidentaux : Betsiboka, Tsiribihina, Manambolo, Menarandra, Mandrare, Onilahy, Fierenana, Mangoky et les petits bassins de la Montagne d'Ambre. Nous la rencontrons également à l'Est, mais uniquement sur la plaine côtière (Ivoloina, Rianila, Namorona, Manampatrana, Matitanana, Manampanihy et quelques petits bassins). Elle partage une partie de la répartition de $S$. madagasca. Sur 91 sites de captures, 32 sont communs avec cette dernière. Mais on observe un net décalage vers les basses altitudes. Sur le versant oriental, $S$. reynae est, à de rares exceptions, cantonnée à la plaine côtière. Sur le versant occidental, elle est fréquente et régulière au-dessous de $1.000 \mathrm{~m}$. Au-dessus, sa présence estr rare et occasionnelle, une situation inverse de celle observée chez $S$. madagasca. $S$. reynae occupe tout le gradient de distance aux sources avec, par rapport à $S$. mádagasca, un léger décalage vers les estuaires. 


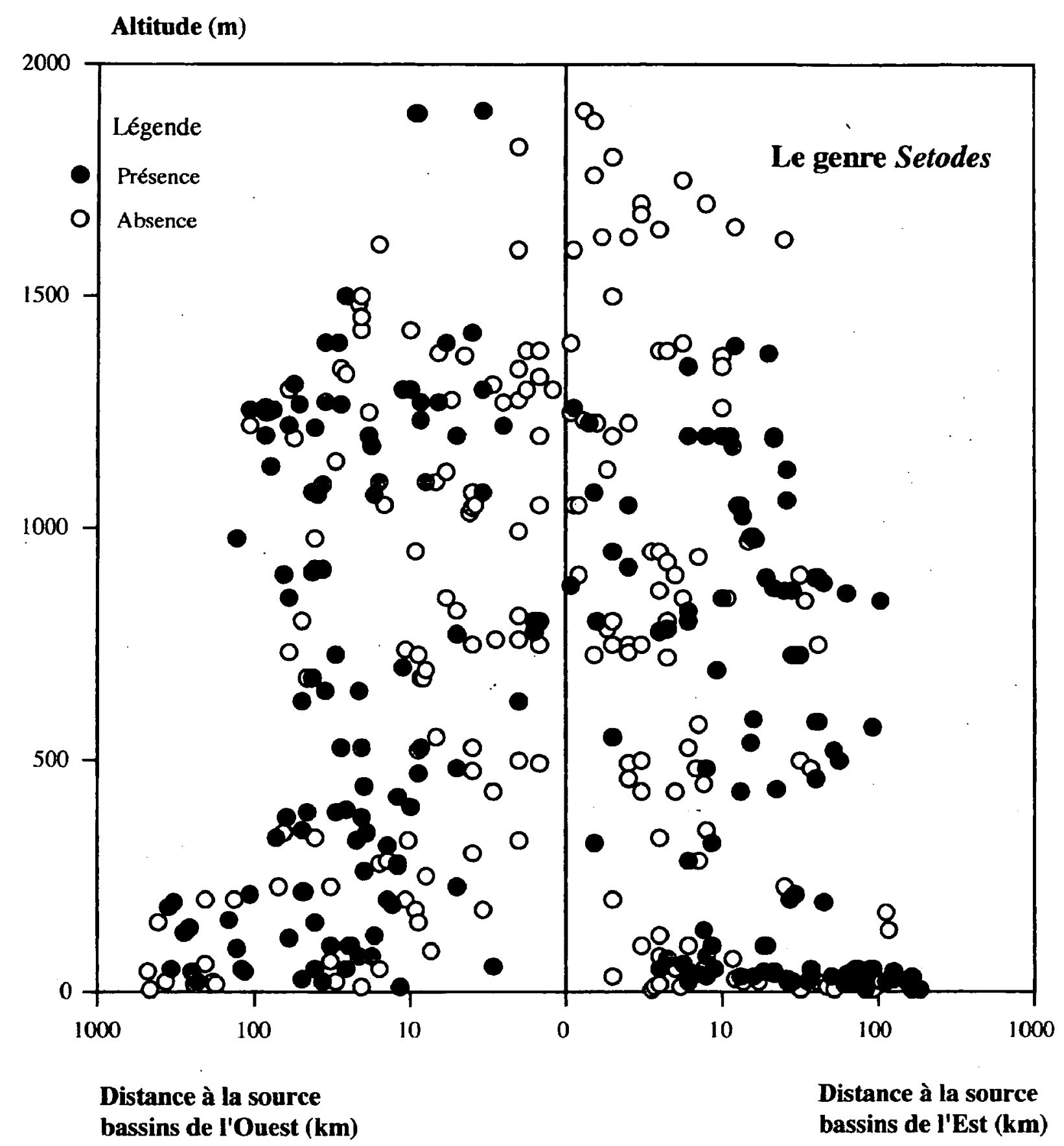

Fig. 26. Profil écologique des captures du genre Setodes.

Fig. 26. Ecological profile, samples of the genus Setodes.

La comparaison des profils écologiques de $S$ reynae et de $S$. madagasca illustre bien la nécessité de distinguer les deux versants. Il apparait, sur chacun d'entreeux, un décalage altitudinal entre les deux espèces. Ces décalages sont dus à la température. Mais il apparaît également, pour chaque espèce, un décalage entre les deux versants qui est dû à leur différence climatique laquelle se traduit par des écarts de température moyenne variant de 2 à $3^{\circ} \mathrm{C}$ (Donque 1972, Chaperon et al. 1993). La figure 29 indique les distributions (valeurs extrèmes, médianes et quartiles) des températures de l'eau, relevées à 18 heures, lors des échantillonnages. C'est une mesure ponctuelle, qui présente trop d'aléas pour remplacer l'altitude dans les profils écologiques. Mais, pour des espèces très fréquentes, elle est utilisable. Nous retrouvons évidemment le décalage entre les deux espèces. Il n'y a, pour $S$. madagasca, aucune différence notable de température entre les sites orientaux et occidentaux. En revanche, dans le cas de $S$. reynae, la population orientale, bien que entièrement située aux basses altitudes, colonise des eaux plus froides que la population occidentale.

- S. fabienneae (Fig. 30) n'est présente que sur le versant occidental, principalement dans le Sud (27 stations sur les bassins suivants : Betsiboka, Mangoky, 

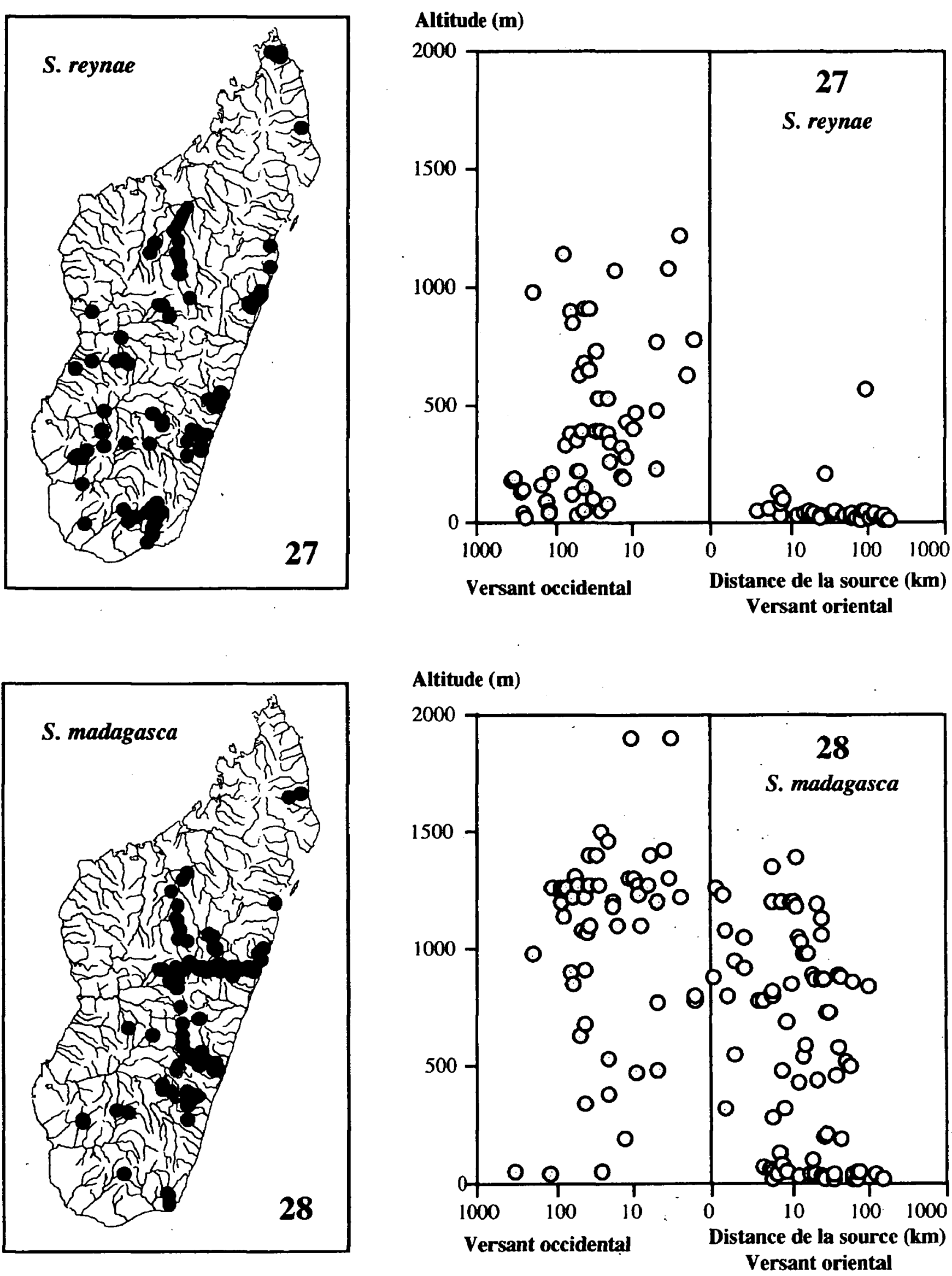

Figs. $27 \& 28$. Localisation et profil écologique des sites de capture de $S$. reynae et $S$. madagasca.

Figs. 27 \& 28. Distribution map and ecological profile, samples of $S$. reynae and $S$. madagasca. 


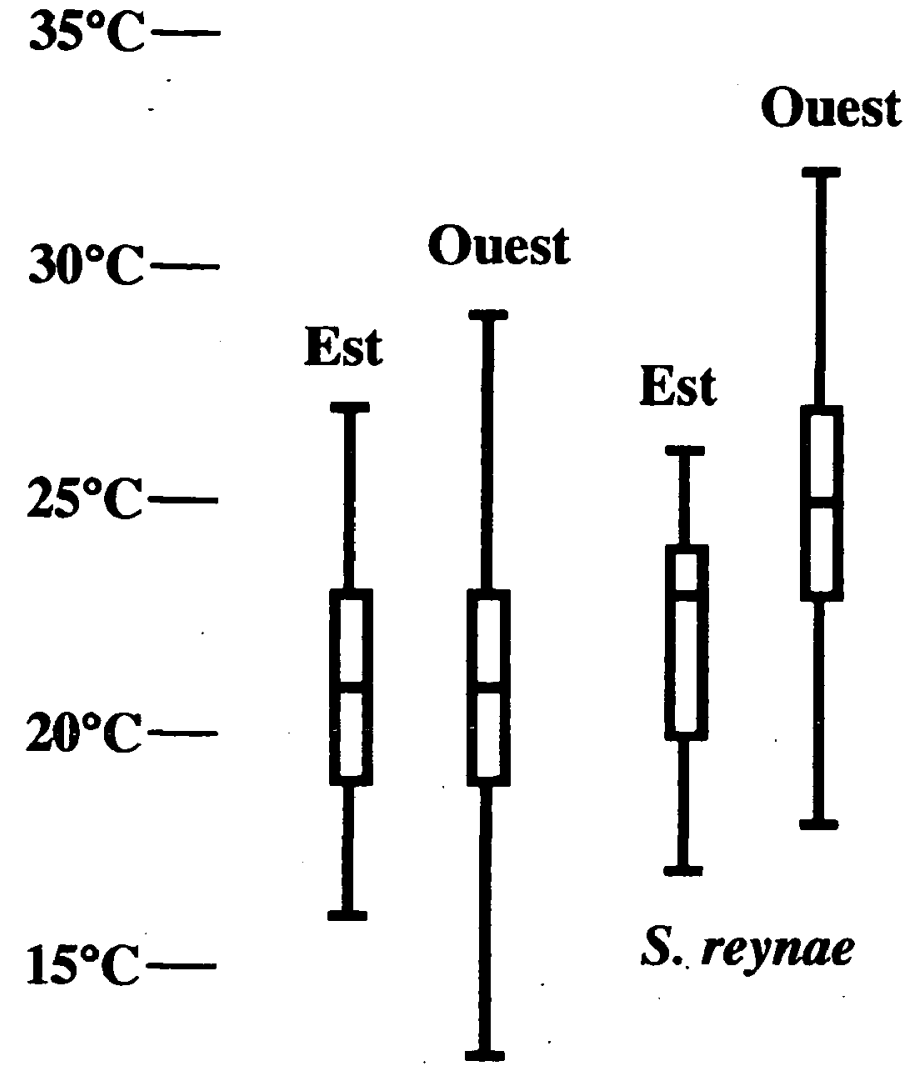

\section{S. madagasca}

\section{$10^{\circ} \mathrm{C}-$}

Fig. 29. Distribution de la température de l'eau à 18 heures sur les sites de capture de $S$. reynae et de $S$. madagasca.

Fig. 29. Distribution of the water temperatures (at 6 p.m.) at the sampling-sites of $S$. reynae and $S$. madagasca.

Tsiribihina, Onilahy, Fierenana, Menarandra, Mandrare et Tarantsy). Elle est cantonnée en-dessous de l'isoligne des $1.000 \mathrm{~m}$, sa distribution est un peu plus centrée sur les rivières moyennes que celle de $S$. reynae.

- Contrairement aux espèces précédentes, $S$. orientalis, est une espèce rare ( 5 stations). Elle colonise des fleuves côtiers ou leurs affluents dans la plaine littorale orientale (Fig. 31, bassins du Rianila, de l'Ivoloina et de la Matitanana). Mais elle est absente des axes principaux qui descendent de l'escarpement et sont très chargés en sédiments. Les quelques captures ont été réalisées dans des milieux stabilisés par des formations secondaires ou des plantations.

- $S$. heryae (11 stations) colonise surtout des milieux lentiques de la côte occidentale (Fig. 31). Elle a été capturée sur les bassins suivants : Betsiboka, Mangoky, Tsiribihina, Fierenana, lac Ihotry et un petit bassin côtier au sud de la Tsiribihina. La moitié des sites de capture sont de petits cours d'eau en situation protégée (des milieux où les eaux sont peu chargées en sédi- ments du fait d'une couverture végétale relictuelle ou secondaire). $S$. heryae est, par exemple, capturée sur les petits ruisseaux aux eaux claires du massif de l'Isalo et, en particulier, dans les zones marécageuses qui en constituent les sources ; les eaux y sont peu profondes, presque stagnantes mais toujours claires. Elle est également présente dans les rares cours d'eau du massif forestier de Zombitse. En revanche, elle est absente des cours qui descendent des Hautes-Terres ou des collines dénudées du Moyen Ouest. Ces localisations écologiques ressemblent à celles de $S$. orientalis. Ces sites sont souvent riches en espèces. Ainsi sur trois de ces stations, nous rencontrons, ensemble, les quatre Setodes de l'Ouest malgache ( $S$. madagasca, reynae, fabienneae et heryae).

\section{Conclusion}

L'ensemble de ces observations nous fournit une bonne approche de l'écologie de ces espèces qu'il faudrait préciser et poursuivre par l'étude des larves. Aucune donnée n'est actuellement disponible sur les larves tropicales. Des élevages ont permis de constater que les Setodes néarctiques s'enfouissent dans les substrats meubles ne laissant en surface que l'ouverture du fourreau. Les contenus stomacaux contiennent aussi bien les sclérites de divers arthropodes que des tissus végétaux ou de fines particules, ce qui laisse supposer un régime varié, composé en grande partie de particules organiques qui sédimentent (Wiggins 1977). Il n'est donc pas étonnant de constater que les Setodes malgaches colonisent souvent les courants calmes, les berges des grandes rivières, voire les milieux lentiques. D'autre part, nous avons noté une faible colonisation des cours supérieurs. Or, ces sites correspondent souvent à des torrents forestiers. Il y a une forte corrélation entre les hautes altitudes, les reliefs accidentés et les vestiges des forêts primaires. Il est, de ce fait, aléatoire d'identifier un facteur limitant; des substrats majoritairement rocheux, des courants vifs, des eaux froides et pauvres en petites particules de matières organiques concourent à éliminer les Setodes de ces milieux.

Deux espèces (madagasca et reynae) sont particulièrement ubiquistes. Elles se distinguent par des exigences thermiques décalées. $S$. madagasca a.un preferendum plus froid (environ trois degrés $\mathrm{C}$ ) que celui de S. reynae.

Les deux espèces rares (orientalis et heryae) préfèrent les milieux chauds. Leur présence coïncide toujours avec des eaux claires dont la présence est due à une couverture végétale stable ou protégée. 

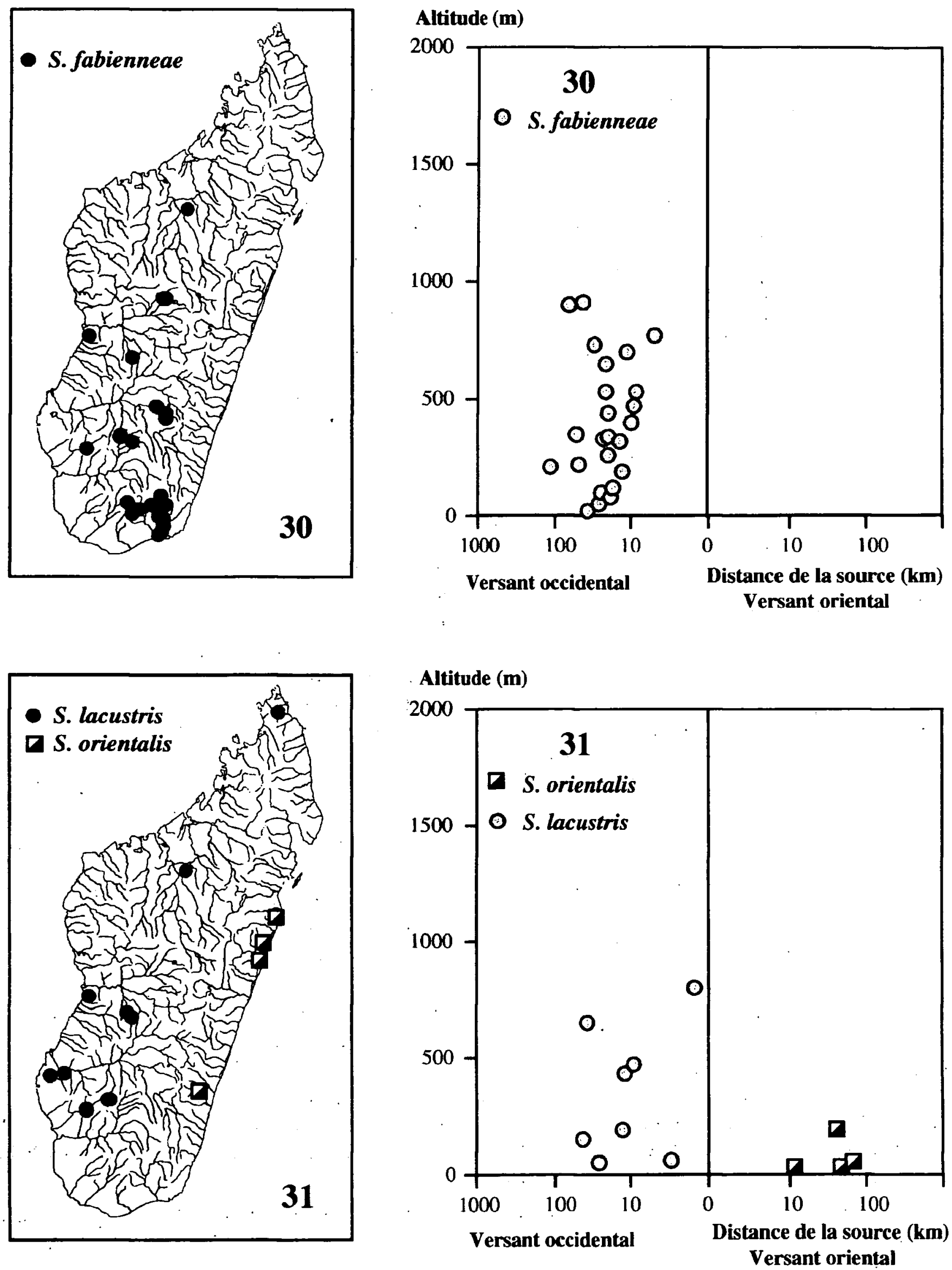

Figs. 30 \& 31. Localisation et profil écologique des sites de capture de S. fabienneáe, S. heryae et S. orientalis. Figs. 30 \& 31. Distribution map and ecological profile, samples of $S$. fabienneae, $S$. heryae and $S$. orientalis. 


\section{Remerciements}

Ce travail constitue la contribution $n^{\circ} 22$ du programme Biodiversité et Biotypologie des eaux continentales malgaches, qui a été mené conjointement par l'Orstom (maintenant IRD) et le CNRE (Antananarivo). Il a bénéficié d'un soutien du Fonds français d'Aide et de Coopération.

\section{Travaux cités}

Battistini R. 1996. - Paléogéographie et variété des milieux naturels à Madagascar et dans les îles voisines : quelques données de base pour l'étude biogéographique de la région malgache. In Biogéographie de Madagascar, Lourenço W.R. (ed.) Orstom, Paris : 1-17.

Beck R.A., Burbank D.W., Sercombe W.J., Riley G.W., Barndt J.V., Berry J.R., Afzal J., Kahn A.M., Jurgen H., Metje J., Chemma A., Shafique N.A., Lawrence R.D. \& Khan M.A. 1995. - Stratigraphic evidence for an early collision between northwest India and Asia. Nature, $373: 55-58$.

Chaperon P., Danloux J. \& Ferry L. 1993. - Fleuves et rivières de Madagascar. Orstom, Paris.

Décamps H. 1968. - Vicariance écologique chez les Trichoptères des Pyrénées. Annls. Limnol., 4 (1) : 1-50.

Donque G. 1972. - The climatology of Madagascar. In Biogeography and Ecology in Madagascar, Battistini R. \& Richard-Vindard G. (eds.), W. Junk, La Haye : 87-144
Gibon F.-M. 2000. - Biologie de la conservation et singularité des cours d'eau. L'exemple des Philopotamidae malgaches (Insecta, Trichoptera). In Diversité et endémisme à Madagascar, Lourenço W.R. \& Goodman S. (eds.) Société de Biogéographie, Paris : 319-330.

Gibon F.-M. \& Elouard J.-M. 1996. - Etude préliminaire de la distribution des insectes lotiques à Madagascar (exemples des Trichoptères Philopotamidae et Diptères Simuliidae). In Biogéographie de Madagascar, Lourenço W.R. (ed.) Orstom, Paris : 507-516.

Mckenzie D.P. \& Sclater J.C. 1973. - The evolution of the Indian Ocean. Sci. American, 228 (5) : 63-72.

Rage J.-C. 1996. - Le peuplement animal de Madagascar : une composante venue de Laurasie est-elle envisageable ? In Biogéographie de Madagascar, Lourenço W.R., (Ed.) Orstom, Paris : 27-35.

Rage J.-C. \& Jaeger J.J. 1995. - The sinking Indian raft : a response to Thewissen and McKenna. Syst. Biol., $44: 260-265$.

Schatz G.E. 1996. — Malagasy / Indo-Australo-Malesian Phytogeographic connections. In Biogéographie de Madagascar, Lourenço W.R. (ed.) Orstom, Paris : 73-83.

Schmid F. 1987. - Considérations diverses sur quelques genres leptocérins (Trichoptera, Leptoceridae). Bull. Inst. r. Sci. nat. Belg. (Entomologie), 57 : 1-147.

Steenis C.G.G.J. van. 1962. - The land-bridge theory in botany. Blumea, $11: 235-372$.

Wiggins G.B. 1977. — Larvae of the North American Caddisfly Genera (Trichoptera). University of Toronto Press, Toronto. 